\title{
Application of Fuzzy Data Fusion in Storage Environment Security Monitoring
}

\author{
Depeng Fan, Shouhai Wang \\ Graduate Department, Beijing Wuzi University, Beijing, China
}

Email address:

714169198@qq.com (Depeng Fan),987633086@qq.com (Shouhai Wang)

To cite this article:

Depeng Fan, Shouhai Wang. Application of Fuzzy Data Fusion in Storage Environment Security Monitoring. American Journal of Applied Mathematics. Vol. 4, No. 5, 2016, pp. 197-203. doi: 10.11648/j.ajam.20160405.11

Received: July 16, 2016; Accepted: July 29, 2016; Published: August 17, 2016

\begin{abstract}
In this paper, fuzzy set theory and multi-sensor data fusion technology are combined and applied in the safety monitoring of the storage environment, this paper establishes the sensor information fusion model and colligate incomplete information collected by multiple sensors, and eliminating the possible redundant and contradictory information among the multi sensor. After the analysis and process of the two level fusion model, the accurate estimation of the safe condition of the storage environment can be obtained. Finally, the method, which can effectively improve the reliability of the safe condition of the storage environment, is verified by an example.
\end{abstract}

Keywords: Multi-sensor Data Fusion, Storage Environment, State Estimation

\section{Introduction}

As a key part of the logistics system, storage plays an important role in the process of transferring, storing and keeping the goods. With the rapid development of social economy and the continuous improvement of people's living standard in recent years, the type of storage goods is increasing, the requirements for storage environment of goods is more and more strict, especially in agricultural products and ordnance etc.

The environmental monitoring of storage mainly refers to the monitoring and management of the storage goods and is an important link in the supply chain. The purpose of monitoring is to timely, accurately and comprehensively reflect the real situation and trend of the monitoring environment [1]. Our traditional storage environment monitoring mainly rely on a single sensor to complete the monitoring of environmental parameters and have heavy workload, information acquisition of a single sensor is easy to be disturbed by the surrounding environment, resulting in a poor data reliability, so that the staff can not accurately obtain the information of the storage environment, so that the accuracy of the system decision-making is affected [2]. Therefore, this paper combines the key parameters of the storage environment and introduces the fuzzy data fusion technology into the storage safety monitoring system, through the real-time monitoring of storage environment, this paper apply multiple sensors to collect the environmental parameters and then combines with fuzzy information processing technology to carry out the data fusion with the collected environmental information, thus the paper can obtain the accurately monitoring information of storage environment and make timely and correct decisions, so that the monitoring environment of the storage is in a stable state.

\section{Multi-sensor Fuzzy Data Fusion Theory}

The definition of fuzzy sets is as follows:

Suppose that a mapping $\mathrm{R}$ is given on the domain $\mathrm{U}$

$$
\begin{aligned}
U & \rightarrow[0,1] \\
u & \rightarrow R(u)
\end{aligned}
$$

$R$ is called fuzzy set of $U, R(U)$ is called membership function of $R$ or $u$ on the membership degree of $R$ [3].

The basic idea of fuzzy sets is to activate the absolute membership of the common set and extend the degree membership of an element to a set from the range of $\{0,1\}$ to the interval $[0,1]$ [4]. In the application of multi-sensor 
information fusion, the membership function of the fuzzy set is used to represent the membership degree of each sensor to the various domains of the environment information, and then the data are dealt by the method of fuzzy transformation to get the accurate state of the environment.

It is assumed that the storage environment monitoring system consists of $\mathrm{N}$ state level, set $\mathrm{U}$ is used to represent the evaluation set of storage environment, the set $\mathrm{V}$ is used to represent factor sets of various sensors for monitoring the storage environment:

$U=\left\{\mathrm{u}_{1} /\right.$ Environmental state $1, \mathrm{u}_{2} /$ Environmental state $2 \ldots \mathrm{u}_{\mathrm{m}}$ /Environmental state $\mathrm{m}\}$

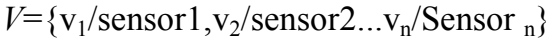

The membership degree of different sensors $v_{i}(i=1,2,3 \ldots n)$ to the environment state $u_{j}(j=1,2,3 \ldots m)$ is $r_{i j}$, the fuzzy relation matrix $R=\left(r_{i j}\right) n \times m$ is constructed by the membership value; in the process of storage environment monitoring, because of the difference of the sensor's performance and the signal strength of the location, the assessment of environmental status is different, so the weight vector is needed. The weights of the environmental monitoring system of different sensors are expressed by $a_{i}(i=1,2, \ldots n)$, it is a fuzzy subset of the sensor factor $\operatorname{set} V$, That is $a_{i}=\mu\left(v_{i}\right)$, the fuzzy vector $A=\left(a_{1}, a_{2}, \ldots, a_{n}\right)$, when $\sum_{i=1}^{n} a_{i}=1, \mathrm{a}_{i} \geq 0, i=(1,2, \ldots \mathrm{n})$ is satisfied, $A=\left(a_{1}, a_{2}, \ldots, a_{n}\right)$ is called factor weight vector, which express the reliability of different sensors to evaluate the environmental state.
When the factor weight vector $A$ of the sensor and the fuzzy relational matrix $R$ are known, the possibility $B$ of storage environment can be obtained by fuzzy transformation.

$$
B=A \bullet R=\left(\mathrm{b}_{1}, \mathrm{~b}_{2}, \cdots \mathrm{b}_{m}\right)=\left(a_{1}, a_{2}, \cdots, a_{n}\right) \bullet\left[\begin{array}{cccc}
r_{11} & r_{12} & \cdots & r_{1 m} \\
r_{21} & r_{22} & \cdots & r_{2 m} \\
\cdots & \cdots & \cdots & \cdots \\
r_{n 1} & r_{n 2} & \cdots & r_{n m}
\end{array}\right]
$$

In the formula, the evaluation operator of fuzzy comprehensive evaluation is shown by the form of ".", each element $b_{j}$ of the $\operatorname{set} B$ is the result the result obtained by the generalized fuzzy synthesis operation, its calculation formula is:

$$
\forall b_{j} \in B, b_{j}=\left(a_{1} \wedge r_{1 j}\right) \vee\left(a_{2} \wedge r_{2 j}\right) \vee \cdots \vee\left(a_{n} \wedge r_{n j}\right), j=1,2, . ., \mathrm{m}
$$

The "and" operation of the generalized fuzzy synthetic operation is" $\wedge$ ", the "or" operation of the generalized fuzzy synthetic operation is " $\vee$ "; Set $B$ is a fuzzy subset of the environmental state set U, Namely $B\left(b_{j}\right)=U\left(u_{j}\right), b_{j}(j=1,2, \ldots, m)$ is the membership of the state of the evaluation of the state of the reviews in the first $j$ after the operation with the combination of the [5]. According to the obtained membership value, people can infer the security status of the storage environment by the certain decision rules, the decision rule is the maximum membership degree principle in this paper.

\section{Structure of Multi-sensor Data Fusion System}

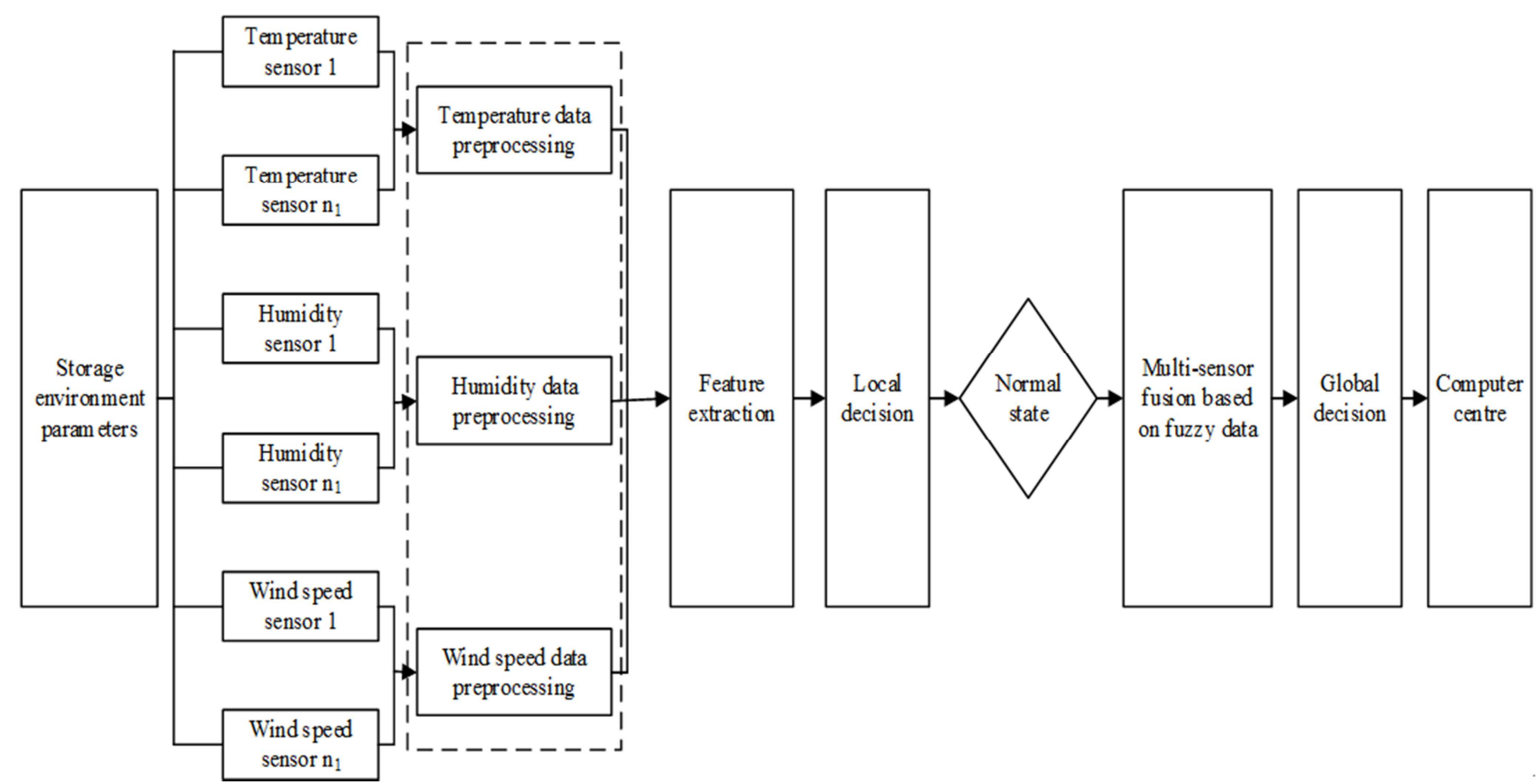

Figure 1. The structure of environmental monitoring system based on multi-sensor fuzzy data fusion. 
The fusion structure of storage environment safety information based on multi-sensors is shown in figure 1. The environmental safety of the storage is related to various environmental parameters. Taking the grain storage as an example, The storage process of grain is related to the factors of environmental temperature, humidity, ventilation rate and air oxygen content whose changes can often reflect the probability of plant diseases and insect pests, which cause the grain mildew [6]. The environmental parameters of grain storage are shown in Figure 2. Fully obtaining the security status of the storage environment by relying on a single sensor can not be done but taking a variety of sensors. Different kinds of sensors are set in different locations in the warehouse. This paper selects three kinds of sensors: temperature sensors, humidity sensors and wind speed sensors. Temperature sensor is used to detect the temperature changes in storage environment, humidity sensor is used to detect the change of humidity in the storage environment; wind speed sensor used to detect the amount of ventilation in storage environment. The fusion structure of Figure 1 is used to detect the state of storage ring.

Fuzzy data fusion is a local decision result of all kinds of sensors, fuzzy data fusion process is on the basis of each local sensor decision, System sequential fusion of the attributes of each sensor decision, finally get the results of global decision. According to the feedback of the results, the computer carry out the process of equipment control. In the system structure, the sensors of temperature, humidity and wind speed monitor three states (normal, slight, dangerous) of the storage. The detected information is often not accurate enough due to the existence of objective factors when the sensors obtain the information. So the data obtained by the sensor must be processed before the data fusion. In this paper, an adaptive weighted fusion algorithm is used to process the raw data. In terms of different sensors, the membership is used to show the possibility of which state the processed information belong to, so it can get a total of 9 membership values of the three groups. According to each membership value, the sensors make local decision at first, if there exists abnormal presence, feedback to the computer center directly. The computer center regulates the environment equipment to enable each environment parameter values to keep within the normal range ; on the contrary, it is necessary to make further second level information fusion, local decision results sent to the fusion center of multi-sensor data fusion based on fuzzy, the result of the global decision is obtained by the fusion calculation . Finally, the decision rule is used to determine the state of the storage environment and the decision results are uploaded to a computer center.

\section{Security Early Warning Model of Storage Environment Based on the Multi-sensor}

\subsection{First Level Preprocessing Based on Adaptive Weighted Fusion Algorithm}

The adaptive weighted fusion algorithm is that the measured value of the monitored data find the corresponding weighted coefficients of the sensor through the self-adaptive method and the optimal fusion result is obtained by weighted fusion.

Under the premise based on the assumption that the data of storage environment monitoring is subject to the uniform distribution, the algorithm can obtain the data fusion value of the total variance according to the data provided by each sensor [7]. Model structure of the self-adaptive weighted fusion algorithm is shown in figure 2 .

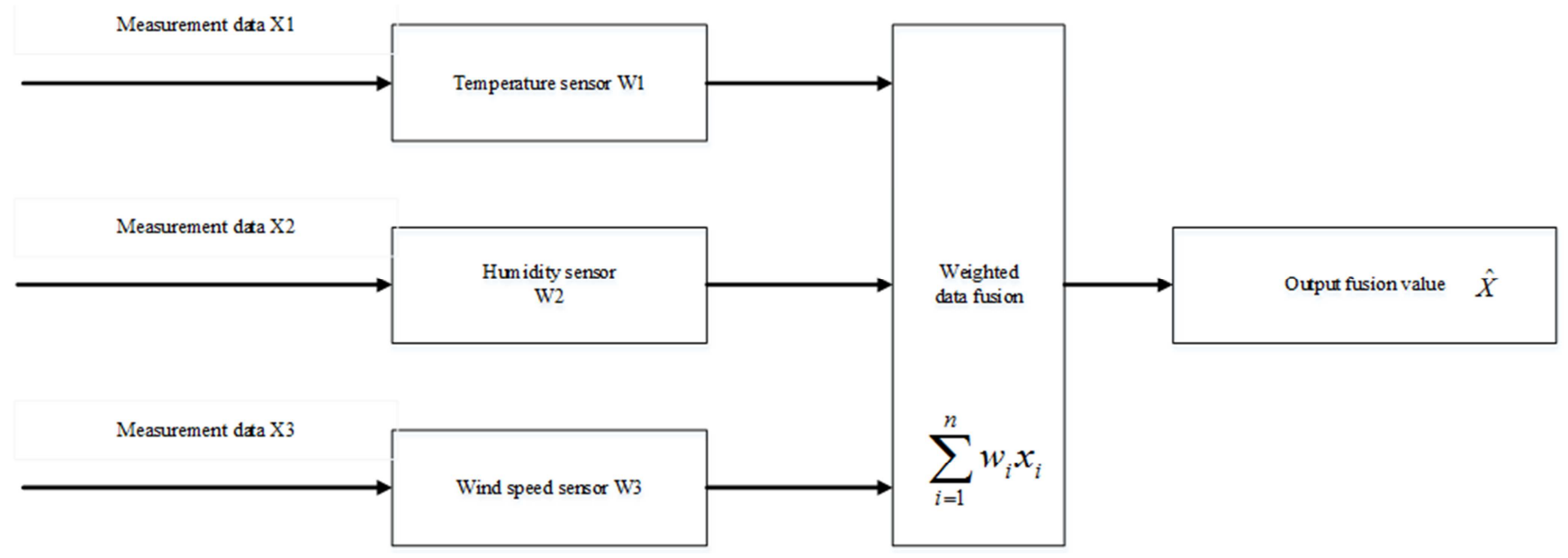

Figure 2. Model structure of the self-adaptive weighted fusion algorithm.

1. The principle of weighted data fusion algorithm

Suppose that in the multi-sensor detection system of storage environment security, there were three groups and every group have $\mathrm{n}$ similar sensors to sample and monitor three parameters of temperature, humidity and wind speed [8]. In the case of temperature, Assuming that the temperature in the storage environment is measured with $\mathrm{n}$ temperature sensor and measured value is $x_{1}, x_{2} \ldots x_{n}$, The variance of each sensor is 
$\Delta_{1}^{2}, \Delta_{2}^{2}, \cdots \Delta_{n}^{2}$, The real value of the temperature to be estimated is $X$, it is suppose that $x_{1}, x_{2} \ldots x_{n}$ is independent of each other and is an unbiased estimate of $X$; Suppose that $w_{1}, w_{2} \cdots w_{n}$ is the corresponding weighting factor of each sensor, and satisfy:

$$
\begin{gathered}
\sum_{i=1}^{n} w_{i}=1 \\
\Delta^{2}=E\left[(\mathrm{X}-\hat{\mathrm{X}})^{2}\right]=E\left[\left(\sum_{i=1}^{n} w_{i} X-\sum_{i=1}^{n} w_{i} x_{i}\right)^{2}\right]=E\left[\left(\sum_{i=1}^{n} w_{i}\left(X-x_{i}\right)\right)^{2}\right]=E\left[\sum_{i=1}^{n} w_{i}^{2}\left(X-x_{i}\right)^{2}+2 \sum_{\substack{i=1, \mathrm{j}=1 \\
i \neq j}}^{n} w_{i} w_{j}\left(X-x_{i}\right)\left(X-x_{j}\right)\right]
\end{gathered}
$$

It is to develop fusion calculation of measured value $x_{i}$ $(\mathrm{i}=1,2, \ldots \mathrm{n}), \hat{X}$ after the fusion needs to meet:

$$
\hat{X}=\sum_{i=1}^{n} w_{i} x_{i}
$$

Because the known $x_{1}, x_{2} \ldots x_{n}$ are independent of each other, and belong to the unbiased estimate of $X$, so:

$$
E\left[\left(X-x_{i}\right)\left(X-x_{j}\right)\right]=0
$$

Then $\Delta^{2}$ can be simplified as:

$$
\left.\Delta^{2}=E\left[\sum_{i=1}^{n} w_{i}^{2}\left(X-x_{i}\right)\right)^{2}\right]=\sum_{i=1}^{n} w_{i}^{2} \Delta_{i}^{2}
$$

$\Delta^{2}$ in the formula (5) is about the multivariate two functions of $w_{1}, w_{2} \cdots w_{n}$, according to the theory of extreme value, there must be a minimum value of total variance $\Delta^{2}$. If the minimum value of the total variance $\Delta^{2}$ is required, it is needed to solve the extreme value of multivariate function under the constraint condition $\sum_{i=1}^{n} w_{i}=1$, that is solution:

$$
\left\{\begin{array}{l}
\Delta^{2}=\min \left(\sum_{i=1}^{n} w_{i}^{2} \Delta_{i}^{2}\right) \\
\sum_{i=1}^{n} w_{i}=1
\end{array}\right.
$$

According to the theory of conditional extreme value based on multivariate function, the optimal weighting factor of the corresponding sensor is obtained:

$$
w_{i}^{*}=\frac{1}{\left(\Delta_{i}^{2} \sum_{i=1}^{n} \frac{1}{\Delta_{i}^{2}}\right)}, i=1,2, \cdots n
$$

At this time, the corresponding total variance is the minimum, the minimum variance is:

$$
\Delta_{\min }^{2}=\frac{1}{\left(\sum_{i=1}^{n} \frac{1}{\Delta_{i}^{2}}\right)}
$$

The principle of weighted data fusion shows that the total variance obtained by the derivation is the estimation of the measured parameters of each sensor at a certain time. But when the estimated true value $X$ is a constant, then people can carry out estimation based on the mean value of the historical measurement data of each sensor in each time, suppose that the measured data of a certain environmental parameters is the $x_{1}, x_{2} \ldots x_{k}$ when a sensor $i$ is located in different times $k$, the mean value of the measured value is :

$$
\bar{x}_{i}(k)=\frac{1}{k} \sum_{j=1}^{k} x_{j}, i=1,2, \cdots n
$$

According to the mean value can be changed the formula (2) as :

$$
\hat{\bar{X}}=\sum_{i=1}^{n} w_{i} \bar{x}_{i}(\mathrm{k})
$$

Then at this time the total variance $\bar{\Delta}^{2}$ is :

$$
\bar{\Delta}^{2}=E\left[(\mathrm{X}-\hat{\overline{\mathrm{X}}})^{2}\right]=E\left[\sum_{i=1}^{n} w_{i}^{2}\left(X-\bar{x}_{i}(\mathrm{k})\right)^{2}+2 \sum_{\substack{i=1, \mathrm{j}=1 \\ \mathrm{i} \neq \mathrm{j}}}^{n} w_{i} w_{j}\left(X-\bar{x}_{i}(\mathrm{k})\right)\left(X-\bar{x}_{j}(\mathrm{k})\right)\right]
$$

Because $x_{1}, x_{2} \ldots x_{n}$ is known to be independent of each other, and meet the unbiased estimate of the $X$, then the mean value $\bar{x}_{1}(\mathrm{k}), \bar{x}_{2}(\mathrm{k}), \cdots \bar{x}_{n}(\mathrm{k})$ of the other sensors also belongs to an unbiased estimate of the $X$, so the formula (11) can be further written:

$$
\bar{\Delta}^{2}=E\left[\sum_{i=1}^{n} w_{i}^{2}\left(X-\bar{x}_{i}(\mathrm{k})\right)^{2}\right]=\frac{1}{k} \sum_{i=1}^{n} w_{i}^{2} \Delta_{i}^{2}
$$

Obviously, the optimal weighting factor $w_{i}^{*}$ obtained by the method of the formula (6) satisfies the formula (12) and 
makes the minimum total variance minimum, at this time the minimum value is:

$$
\bar{\Delta}_{\min }^{2}=\frac{1}{\mathrm{k}\left(\sum_{i=1}^{n} \frac{1}{\Delta_{i}^{2}}\right)}=\Delta_{\min }^{2} / k
$$

Formula (13) shows that the total error of $\bar{\Delta}_{\min }^{2}$ is smaller than that of $\Delta_{\min }^{2}$, and $\bar{\Delta}_{\min }^{2}$ decreases with the increase of $\mathrm{k}$. Therefore, the weighted fusion method can improve the accuracy of the estimated value.

2. The adjustment of the weighted dynamic factor

But in the formula (5), the optimal weighting factor $w_{1}, w_{2} \cdots w_{n}$ is determined by the variance $\Delta_{i}^{2}(\mathrm{i}=1,2, \cdots \mathrm{n})$ of each sensor. Currently in the practical application, variance of acquisition does not consider environmental factors in most cases, but is directly determined by its variance parameters of the sensor device or specified by the experience, such shortcomings can not reflect the uncertainty of the measurement values.

Because the variance of acquisition can't better reflect the uncertainty of the data, so people can use the following ways to obtain the sensor variance.

By the hypothesis of formula (9), people has obtained the mean value of the measured data of a certain sensor $\mathrm{i}$ at $\mathrm{k}$ different moments, so the variance of the measured values for $\mathrm{k}$ is:

$$
\Delta_{k}^{2}=\frac{1}{k} \sum_{i=1}^{k}\left(x_{i}-\bar{x}_{k}\right)^{2}
$$

Assuming the measurement data of the first $k+1$ time is $x_{k+1}$, according to the deduction of mean formula of the first $k$ measured data, and the mean value formula for the first $k+1$ measurement data can be obtained:

$$
\bar{x}_{i}(k+1)=\frac{1}{k+1} \sum_{j=1}^{k+1} x_{j}=\frac{1}{k+1}\left(\sum_{j=1}^{k} x_{j}+x_{k+1}\right)=\frac{1}{k+1}\left(k \bar{x}_{k}+x_{k+1}\right)
$$

The formula (14) shows that the variance of the $k+1$ measured values is:

$$
\Delta_{k+1}^{2}=\frac{1}{k+1} \sum_{i=1}^{k+1}\left(x_{i}-\bar{x}_{k+1}\right)^{2}
$$

The formula (15) is brought into the formula (16), and according to the variance of the first $k$ measurement data, people can make the deduction of formula (16) as follow:

$$
\Delta_{k+1}^{2}=\frac{1}{k+1} \sum_{i=1}^{k+1}\left(x_{i}-\bar{x}_{k+1}\right)^{2}=\frac{1}{k+1} \sum_{i=1}^{k+1}\left[x_{i}-\frac{1}{k+1}\left(k \bar{x}_{k}+x_{k+1}\right)\right]^{2}=\frac{1}{k+1} \sum_{i=1}^{k+1}\left[x_{i}-\bar{x}_{k}+\frac{1}{k+1}\left(\bar{x}_{k}-x_{k+1}\right)\right]^{2}
$$

At this moment, so $\alpha=\frac{1}{k+1}\left(\bar{x}_{k}-x_{k+1}\right)$, then people can continue the deduction of the formula (17):

$$
\begin{aligned}
& \Delta_{k+1}^{2}=\frac{1}{k+1} \sum_{i=1}^{k+1}\left[\left(x_{i}-\bar{x}_{k}\right)+\alpha\right]^{2}=\frac{1}{k+1} \sum_{i=1}^{k+1}\left[\left(x_{i}-\bar{x}_{k}\right)^{2}+2 \alpha\left(x_{i}-\bar{x}_{k}\right)+\alpha^{2}\right]=\frac{1}{k+1} \sum_{i=1}^{k+1}\left(x_{i}-\bar{x}_{k}\right)^{2}+\frac{1}{k+1} \sum_{i=1}^{k+1} 2 \alpha\left(x_{i}-\bar{x}_{k}\right)+\frac{1}{k+1} \sum_{i=1}^{k+1} \alpha^{2} \\
& =\frac{1}{k+1}\left[k \Delta_{k}^{2}+\left(x_{k+1}-\bar{x}_{k}\right)^{2}\right]+\frac{2 \alpha}{k+1} \sum_{i=1}^{k+1} x_{i}-2 \alpha \bar{x}_{k}+\alpha^{2}=\frac{k}{k+1} \Delta_{k}^{2}+\alpha\left(\bar{x}_{k}-x_{k+1}\right)+2 \alpha \bar{x}_{k+1}-2 \alpha \bar{x}_{k}+\alpha^{2}=\frac{k}{k+1} \Delta_{k}^{2}+\alpha\left(\bar{x}_{k}-x_{k+1}\right)-\alpha^{2}
\end{aligned}
$$

To bring $\alpha=\frac{1}{k+1}\left(\bar{x}_{k}-x_{k+1}\right)$ into the formula (18),

$$
\Delta_{k+1}^{2}=\frac{k}{k+1} \Delta_{k}^{2}+\alpha\left(\bar{x}_{k}-x_{k+1}\right)-\alpha^{2}=\frac{k}{k+1} \Delta_{k}^{2}+\frac{k}{(k+1)^{2}}\left(\bar{x}_{k}-x_{k+1}\right)^{2}
$$

Formula (19) is the recurrence formula of the variance of $\mathrm{k}+1$ measurement data. The recurrence formula can be used to calculate the variance of the real-time measurement data. When the calculated variance is brought into the formula (7), people can obtained the weighted value of each sensor and obtain the corresponding fusion value of each sensor.

Through the fusion processing of the stage, the influence brought by the abnormal data can be effectively reduced, people can get relatively accurate data of storage environment parameters and make the local decision of the same type of sensor. According to the results of the local decision to determine the safe state of environment, if the state of the environment is abnormal, people can go into the Environmental Monitoring Center of storage to carry out early warning and take corresponding safety measures according to the warning level; if the state of the environment is normal, people need the further fusion processing.

\subsection{Decision Level Information Fusion Based on Fuzzy Theory}

\subsubsection{Membership Function Seek Fuzzy Relation Matrix $R$}

The fuzzy relation matrix is constructed by the membership degree of different kinds of sensors to the environment state, but the degree of membership must be determined by 
membership function [9]. That is to say, to determine the fuzzy relation matrix, people should determine the form of the membership function which is the characteristic value of each sensor relative to the state of the storage environment. The state of the storage environment is divided into three kinds of fuzzy state, namely: normal, slight and dangerous, $i, j$ respectively denote the different sensors and different states of the environment, according to the observed experimental data, the membership function $r_{i j}(x)(i=1,2,3 ; j=1,2,3)$ is defined by Cauchy type distribution.

The membership function can be expressed as:

$$
\begin{gathered}
r_{i 1}(\mathrm{x})=\left\{\begin{array}{l}
1, x_{i} \leq a_{i 1} \\
\frac{1}{1+\alpha\left(\mathrm{x}_{i}-\mathrm{a}_{i 1}\right)^{\beta}}, x_{i}>a_{i 1}
\end{array}(\alpha>0, \beta>0)\right. \\
r_{i 2}(x)=\frac{1}{1+\alpha\left(x_{i}-a_{i 2}\right)^{\beta}}(\alpha>0, \beta>0 \text { and is even }) \\
r_{i 3}(\mathrm{x})=\left\{\begin{array}{l}
0, x_{i} \leq a_{i 3} \\
\frac{1}{1+\alpha\left(\mathrm{x}_{i}-\mathrm{a}_{i 3}\right)^{-\beta}}, x_{i}>a_{i 3}
\end{array}(\alpha>0, \beta>0)\right.
\end{gathered}
$$

In the membership function $r_{i 1}, r_{i 2}, r_{i 3}$ are the membership value of three states (include normal, mild, dangerous) about the storage environment which is determine by sensor $v_{i} ; x_{i}$ indicates the actual eigenvalue that the sensors obtain; $a_{i 1}, a_{i 2}, a_{i 3}, a_{i 4}$ is the standard eigenvalue of three storage environment states which are determine by sensor $i$. According to the $r_{i j}$, the fuzzy relation matrix $R$ can be obtained, and then the relation matrix $R^{*}=\left\{r_{i j}^{*}\right\}$ can also be obtained after non-dimensional sequences.

\subsubsection{Analytic Hierarchy Process Fuzzy Vector}

Fuzzy vector $A$ is the fuzzy subset of sensor factors set $\mathrm{V}$ and formed by the weight of the environment monitoring system of different sensors.

The sensor weight $a_{i}$ is determined by expert evaluation method while "Storage environmental safety factors index weight table" will be provided for the expert [10].

Table 1. Expert judgment matrix is determined.

\begin{tabular}{llll}
\hline $\mathbf{V} \rightarrow \mathbf{V}_{\mathbf{i}}$ & $\mathbf{V}_{\mathbf{1}}$ & $\mathbf{V}_{\mathbf{2}}$ & $\mathbf{V}_{\mathbf{3}}$ \\
\hline $\mathrm{V}_{1}$ & & & \\
$\mathrm{~V}_{2}$ & & & \\
$\mathrm{~V}_{3}$ & & & \\
\hline
\end{tabular}

According to the evaluation expert, the factor weight of each sensor can be obtained by APH (analytic hierarchy process), then people can get the fuzzy vector $A^{*}=\left(a_{1}{ }^{*}, a_{2}{ }^{*}, a_{3}{ }^{*}\right)$ after non-dimensional sequences.

\subsubsection{Synthesis Algorithm and Rules}

In the synthetic operation of the fuzzy data fusion center, the synthesis result of the sensor weight vector $A^{*}$ and the fuzzy relation matrix $R^{*}$ is the fuzzy subset of the evaluation set $\mathrm{U}$, and the synthesis algorithm based on the fuzzy set theory is obtained:

$$
B=A^{*} \bullet R^{*}=\left(\mathrm{b}_{1}, \mathrm{~b}_{2}, \mathrm{~b}_{3}\right)=\left(a_{1}{ }^{*}, a_{2}{ }^{*}, a_{3}{ }^{*}\right) \bullet\left[\begin{array}{lll}
r_{11}{ }^{*} & r_{12}{ }^{*} & r_{13}{ }^{*} \\
r_{21}{ }^{*} & r_{22}{ }^{*} & r_{23}{ }^{*} \\
r_{31}{ }^{*} & r_{32}{ }^{*} & r_{33}{ }^{*}
\end{array}\right]
$$

Synthetic operation results $B=\left(b_{1}, b_{2}, b_{3}\right)$ is membership degree value about storage security state (normal, mild, or dangerous) after the data fusion of three different sensors in the process of storage environment condition monitoring, according to the calculation result, the final global decision is determined by the method based on rules. Rule based method of decision rule is [11]:

(1) the target state should have the maximum membership value;

(2) the membership value of target state is greater than a certain threshold, such as 0.50 ;

(3) the difference of membership value between the target state and the other state is greater than a single threshold, such as 0.10 .

\section{Case Analysis}

In a food storage environmental safety monitoring system, using temperature sensors, humidity sensors and wind speed sensors to collect the environment information of multiple locations in the storage environment. According to the above analysis, a collection of sensor: $V=\left\{v_{1}, v_{2}, v_{3} /\right\}=$ \{temperature sensor, humidity sensor, speed sensor\}, the set of the environment state $U=$ \{normal, mild, dangerous . Table 1 gives the storage environment in different states, storage environmental parameters measured at the sensor set, set the state of the environment, measured environmental parameters of the membership values (normalized value), according to experience given by expert, and using the analytic hierarchy analysis method to get the weight of each sensor and the result is calculated after fusion on the basis of the theoretical analysis above.

Table 2. Comparison of the results of fuzzy data fusion and single sensor identification based on multi-sensor.

\begin{tabular}{llllll}
\hline Index weight vector $\left(\mathbf{A}^{*}\right)^{\mathbf{T}}$ & Environmental state & normal & slight & danger & Monitoring results \\
& Sensor & $\begin{array}{l}\text { Actual state of } \\
\text { storage environment }\end{array}$ \\
\hline 0.34 & Temperature sensor $\mathrm{v}_{1}$ & 0.513 & 0.497 & 0 & unknown \\
0.36 & humidity sensor $\mathrm{v}_{2}$ & 0.842 & 0.158 & 0 & normal \\
0.3 & Wind speed sensor $\mathrm{v}_{3}$ & 1 & 0 & 0 & normal \\
$\mathrm{A}^{*} \cdot \mathrm{R}^{*}$ & Fusion calculation & 0.777 & 0.224 & 0 & normal \\
0.34 & Temperature sensor $\mathrm{v}_{1}$ & 0.245 & 0.638 & 0.117 & slight \\
\hline
\end{tabular}




\begin{tabular}{|c|c|c|c|c|c|c|}
\hline Index weight vector $\left(A^{*}\right)^{T}$ & $\begin{array}{ll}\text { Sensor } & \text { Environmental state } \\
\end{array}$ & normal & slight & danger & Monitoring results & $\begin{array}{l}\text { Actual state of } \\
\text { storage environment }\end{array}$ \\
\hline 0.36 & humidity sensor $v_{2}$ & 0.124 & 0.784 & 0.092 & slight & slight \\
\hline 0.3 & Wind speed sensor $v_{3}$ & 0.576 & 0.424 & 0 & normal & \\
\hline$A^{*} \cdot R^{*}$ & Fusion calculation & 0.301 & 0.626 & 0.073 & slight & \\
\hline 0.34 & Temperature sensor $\mathrm{v}_{1}$ & 0 & 0.221 & 0.779 & serious & \\
\hline 0.36 & humidity sensor $\mathrm{v}_{2}$ & 0 & 0.108 & 0.892 & serious & danger \\
\hline 0.3 & Wind speed sensor $v_{3}$ & 0 & 0 & 1 & serious & \\
\hline
\end{tabular}

Table 1 shows the results of three sensors' (temperature, humidity and wind speed sensors) monitoring the status of the storage environment individually and the results of the fusion of multiple sensors. According to the results, we learn that sometimes the monitoring results may be inconsistent with the actual state of the environment when the sensor works separately, for example, when the actual state of the storage environment is at the state of slight, the wind speed sensor separately shows that it is normal, and the results show that it is slight according to the multi-sensors fusion calculation. Therefore, according to the use of multi-sensor fuzzy fusion method, the monitoring results reflect the state of the storage environment more accurately and more safely, the limitations of single sensor itself of false positives and misstatement are greatly reduced, and the accuracy rate of the warehouse environment monitoring is also improved.

\section{Conclusion}

In storage environmental safety monitoring system, for the granary, the three environmental parameters of temperature, humidity, ventilation have an important influence on food safety storage, so it is necessary to monitor the three environmental parameters in real time in the storage environment safety monitoring. In this paper, fuzzy set theory and multi-sensor data fusion technology are combined to give the fusion structure and method which is also applied in the storage environment security monitoring. Through the empirical analysis, it is proved that the fuzzy data fusion method based on multi-sensors is more accurate than the single sensor to monitor the state of the storage environment, and it also improve the reliability of the storage environment monitoring system effectively.

\section{References}

[1] Zhang Qiu. Research on the Application of Wireless Sensor
Network in Storage Environment Monitoring [D]. Jiangnan University, 2013.

[2] Liu Yachuang. TheResearch of Underwater Sensor Networks Deployment Based on Data Fusion [D]. Henan University of Science and Technology, 2015.

[3] Xie Jijian, Liu Chengping. The Method of Fuzzy Mathematics and its Application [M]. Wuhan: Huazhong University of Science and Technology Press, 2014.

[4] Zhang Mingyang, Research of WSN-based Data Fusion in Water Quality Monitoring [J]. Computer engineering and application, 2014, 23: 234-238+260.

[5] Liu Dong, Liu Dejun. Multi-sensor Integration and Information Fusion and its Application Research [J]. Chemical engineering and equipment, 2015, 05: 170-171+213.

[6] Chu Qianyun, Chen Hao, Liao Tongqing. Remote Intelligent Methane Tank Monitoring System Based on Multi-sensor Information Fusion Technology [J]. Computer knowledge and technology, 2016, 10: 234-236.

[7] Zhai Weiqing, Zhao Zhimin. Application of Multi-sensor Information Fusion Method and CAN Bus Technology in Coal Mine Safety Environment Monitoring [J]. Science \& Technology vision, 2015, 20: 16.

[8] Yi Gao Xiang, Pan Changcheng, Guo Jianzhong, Wang Shibin, Wang rujun, Kang Rongxue. Study on Safety Monitoring Model of Petroleum Tank Farm Based on Multi-source Data Fusion [J]. China safety science and technology, 2014, 03: 90-94.

[9] Liu Qian.Agriculture IOT-oriented Multi-environment Information Fusion for Monitoring and Recognition [D]. Donghua University, 2014.

[10] Chen Qian. Design and Implementation of WSN Based on Data Fion for Environment Monitoring in Communication Equipment Room [D]. Chongqing University, 2014.

[11] Zhao Juanni. Multi-sensor Data Fusion Technology and its Application in Photovoltaic Power Plant Monitoring System [J]. Science and technology information, 2013, 07: 401-402. 